\title{
BMJ Open Quality Improving referral to psychological support unit at Saudi Red Crescent Authority in Riyadh Region
}

\author{
Ahmad Yousif Alzahrani, ${ }^{1}$ Sabri abd Allah Mahmod, ${ }^{1}$ \\ Thamer Mohammad Bakhamis, ${ }^{1}$ Khaled Al-Surimi ${ }^{2,3}$
}

To cite: Alzahrani AY, Mahmod SaA, Bakhamis TM, et al. Improving referral to psychological support unit at Saudi Red Crescent Authority in Riyadh Region.BMJ Open Quality 2017;6:e000089. doi:10.1136/ bmjoq-2017-000089

Received 18 April 2017 Revised 20 September 2017 Accepted 22 September 2017

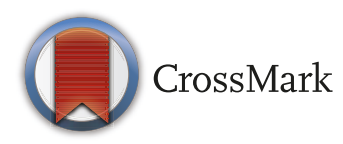

${ }^{1}$ Department of Emergency Medical Services, Saudi Red Crescent Authority, Riyadh, Saudi Arabia

${ }^{2}$ Department of Health System and Quality Management, King Saud bin Abdulaziz University for Health Sciences, College of Public Health and Health Informatics, Riyadh, Saudi Arabia

${ }^{3}$ Department of Primary Care and Public Health, Imperial College, London, UK

Correspondence to Dr Ahmad Yousif Alzahrani; ahmad96@hotmail.com

\section{ABSTRACT}

The Psychological Support Unit (PSU) performance in Saudi Red Crescent Authority (SRCA) showed that only a small number of case referred seeking psychological advice and management from PSU among all SRCA employees. However, research shows that between $28 \%$ and $52 \%$ of emergency medical services (EMS) providers usually seek psychological help in various EMS cultures, where $86 \%$ of them usually suffer from critical stress. Thus, we decided to design a quality improvement project that aims to improve the referral process by increasing cases referred to the PSU at SRCA in Riyadh Region by $75 \%$ in 2 months. A multidisciplinary team has been formed to analyse the problem using quality tools including, brainstorming, fishbone diagram and flow chart of the PSU processes. Several possible reasons have been identified, such as lack of awareness among the SRCA's employees about PSU and its services, and the concern about privacy and confidentiality during psychological consultations in the PSU, in addition to the long referring process to PSU. The team decided to test the following change ideas: increasing the awareness of employees about the PSU services, improving the privacy and confidentiality during the consultation using electronic channels, and finally re-engineering the referral process to make it lean and remove all the unnecessary steps. Several improvement interventions have been tested sequentially in three consecutive Plan-Do-Study-Act cycles on a weekly basis. The project findings demonstrated that the first change idea was successful but not reaching the target while the second change had led to huge impact exceeding our target but with short effect. On the other hand, although the third change idea of re-engineering the PSU referral process had led to negative result initially, over the following weeks of measurement the results turned to be positive and meeting our expectations. We concluded that re-engineering referral process is most effective improvement intervention among other change ideas in term of magnitude and sustainability of the effect on increasing the number of referral cases to the PSU. We recommend conducting further testing and measuring of these change ideas in other PSU across the SRCA to understand the diffident context in other regions of SRCA.

\section{PROBLEM}

The Saudi Red Crescent Authority (SRCA) is the main provider of prehospital emergency care across the Kingdom of Saudi Arabia. In order to maintain a good mental health for its employees, the SRCA has established the Psychological Support Unit (PSU) in the main office in Riyadh 5 years ago. The PSU aims at providing psychological support, prevention and treatment of different psychological conditions among emergency medical services (EMS) providers. However, the data analysis of the PSU showed that only a small number of referred cases seek psychological advice and management as compared with the overall number of staff working in SRCA. The average number of visits per month to PSU is 2.5 visits, ranging between 0 and 4 (see figure 1). These numbers of visits are very low in comparison with total number of more than 10000 employees at SRCA. On the other hand, research shows that between $28 \%$ and $52 \%$ of EMS providers usually seek psychological help in various EMS cultures, where $86 \%$ of them usually suffer from critical stress. Thus, we decided to design a quality improvement project that aims to improve the referral to the PSU at SRCA in Riyadh Region by $75 \%$ in 2 months.

\section{BACKGROUND}

Psychological and mental health problems among people working in EMS are expected, especially those at the front-line staff in the field. A survey was conducted in 2015 for a total of 4021 EMS providers in USA named 'What's Killing Our Medics?' to evaluate the stress and the working environment for paramedics. The survey revealed high levels of stress, suicide thoughts and attempts. Some participants found that the support units are effective, but still there is area for improvement, besides the culture that is not supportive. ${ }^{1}$ Likewise, Newland et al have reported that EMS providers seeking psychological help for stress in various EMS cultures in the USA range from $28 \%$ to $52 \%$, where $86 \%$ of them suffered critical stress. ${ }^{2}$ A study in the Netherlands revealed that most of the 


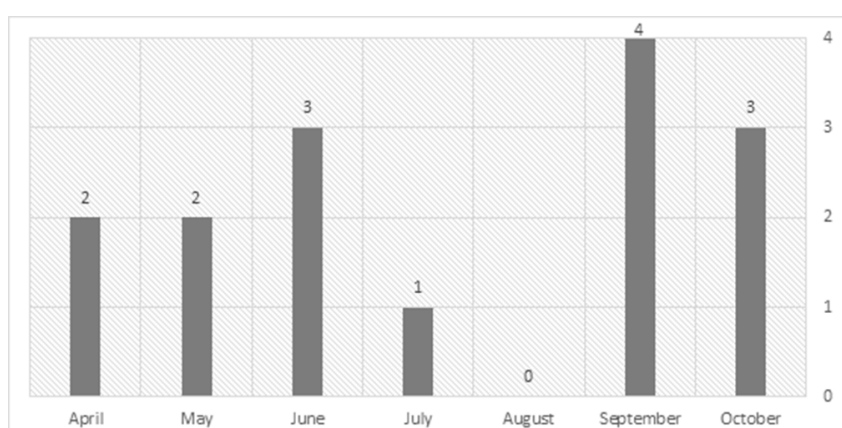

Figure 1 Number of new cases referred to the Psychological Support Unit at Saudi Red Crescent Authority in 2016.

ambulance employees had faced acute stressors in their work. ${ }^{3}$ More specifically a survey on ambulance workers in UK had also shown that the rate of post-traumatic stress disorder was $22 \%$. Almost $10 \%$ reported suspected depression and $22 \%$ suspected anxiety based on the Hospital Anxiety and Depression Scale scores. ${ }^{4}$ Burnout, as one of the consequences of emergency medicine, was reported in high levels among physicians working in a hospital. ${ }^{5}$ Poor sleep quality and fatigue were found to be common and associated with safety outcomes among EMS providers. ${ }^{6}$ Recently, Ketelaar et $a l^{7}$ have proposed innovative-based technology approach using electronic email for mental health testing among nurses and allied health professionals and showed potential impact on the work functioning and mental health of the participants. ${ }^{7}$ Furthermore, a randomised controlled trial has proven that internet-based cognitive behaviour therapy was effective in reducing depressive symptoms in the experimental

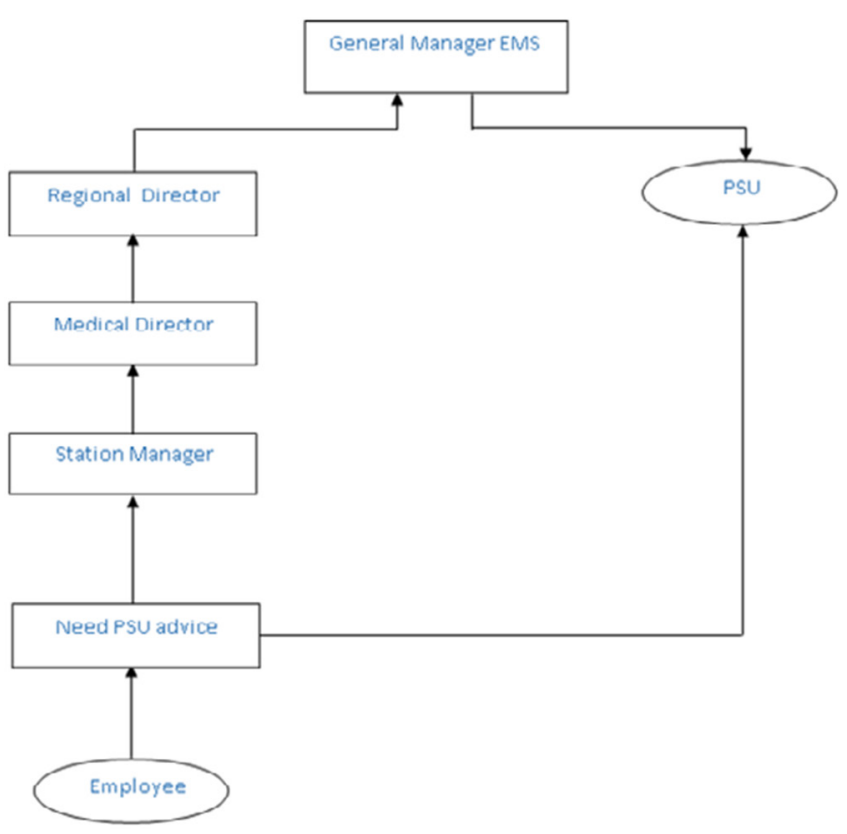

Figure 3 Flow chart of referral process. EMS, emergency medical services; PSU, Psychological Support Unit.

group. ${ }^{8}$ A call for interdisciplinary interventions for EMS workers has been brought to light in the form of mental wellness programme due to the complexity of this job. ${ }^{9}$

\section{BASELINE MEASUREMENT}

The baseline data showed that the number of cases referred to PSU ranges from 0 to 1 per week, that is four cases per month at most for the last 6 months period of 2016, with an average of 2.5 cases per month, as shown

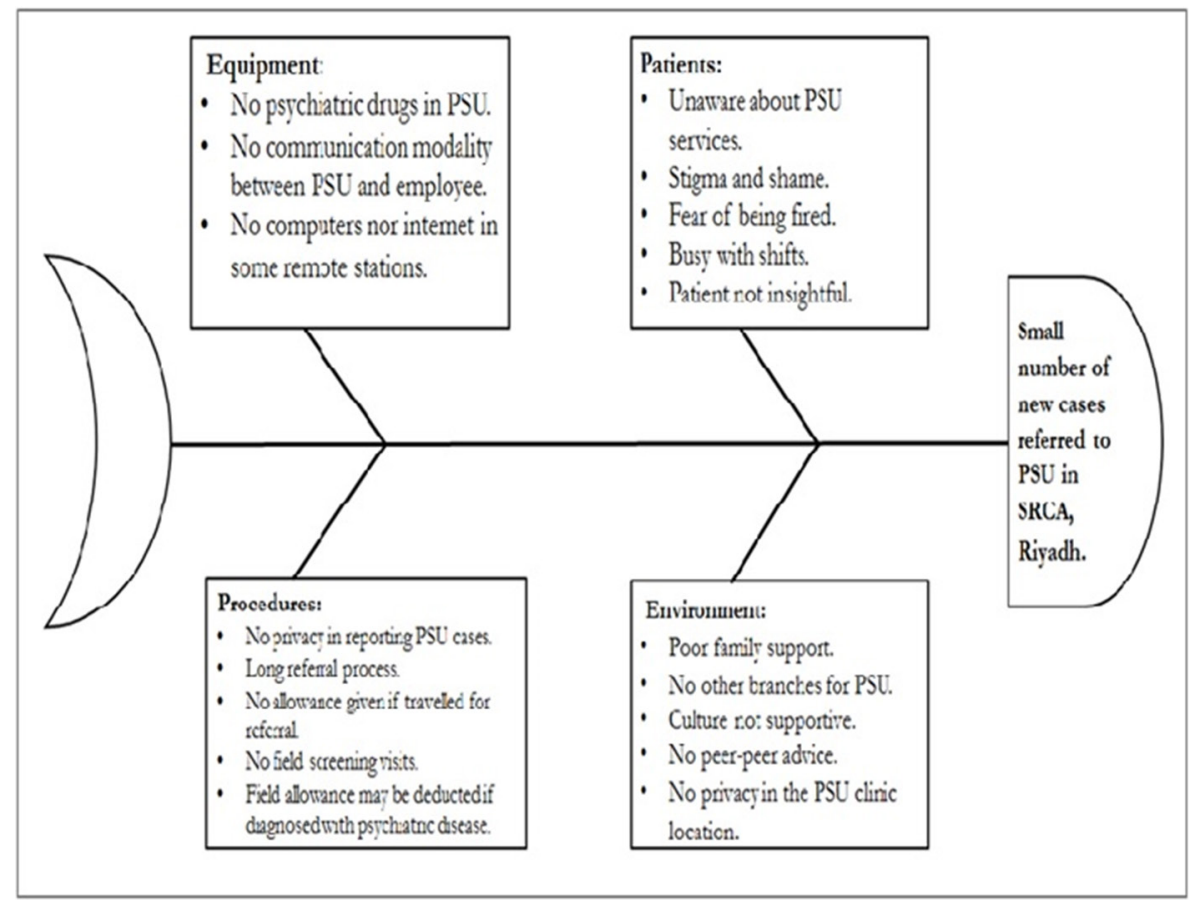

Figure 2 Fishbone analysis used for root cause analysis. PSU, Psychological Support Unit; SRCA, Saudi Red Crescent Authority. 
in figure 1 . We used the absolute number of new cases referred per week to PSU in SRCA Riyadh as outcome measure by calculating the number of referral cases utilising patients' records on a weekly basis. In addition, we used two process measures related to the outcome measures, first the number of electronic consultations conducted in PSU weekly and documented in patients' records. The data have been collected and reviewed by an assigned psychiatrist in the quality team. The second process measure is the percentage of cases referred directly to PSU in SRCA as a result of re-engineering the PSU process calculated and extracted on a weekly basis from patients' records.

\section{DESIGN}

A multidisciplinary team had been formed consisting of a team leader, a psychiatrist, a psychologist, a station manager, an information technologist, a general director of EMS department and an emergency medical technician. The team started by analysing the causes of problem using different quality tools, including brainstorming technique, cause and effect using fishbone diagram (figure 2), and flow chart (figure 3) of the PSU processes, among others. Initially the team highlighted the flow chart of the current referral process and conducted root cause analysis to better analyse the causes of the problem. Lack of awareness, minimal confidentiality and long referral route were the important causes of the small number of cases referred due to the lack of sufficient data that can help in selecting the appropriate change ideas; the team relies solely on their work experience and hunches in selecting the change ideas. The team member agreed on the following: raising the awareness of PSU existence and the services provided among all the SRCA staff by sending brochure through emails to all SRCA staff. Another proposed intervention is establishing electronic consultation channels that guarantee privacy and confidentiality during the psychological consultations. Lastly, a change to deal with the long complicated referral process was an important one, so the team suggested re-engineering the referral process to make it short and smooth, making sure the services of PSU are accessible for all. The Model For Improvement was used as quality method to test the effect of these selected changes by conducting sequential Plan-Do-Study-Act cycles 1 week for each change.

\section{STRATEGY}

\section{Plan-Do-Study-Act 1}

Sending PSU identifying brochures through SRCA emails to the employees in order to raise awareness about PSU services which might improve the referral process to the unit. A psychologist was assigned to this task, which was on Sunday, November 20. The run chart shows the initial baseline of the referred cases to the unit, ranging from 0 to 1 for the last 9 weeks.
Furthermore, the number of new cases distributed in relation to the median and target line, which is measured on a weekly basis, is shown. As we can observe, after conducting Plan-Do-Study-Act 1 in week 10, we get one case, which is on our median but still below our target. Hence, only mild improvement was observed, so we decided to add the second change idea of establishing electronic consultation channels for PSU (see more details about PDSA 1 in table 1 ).

\section{Plan-Do-Study-Act 2}

Establishing electronic consultation channels for employees seeking support, which allow more confidential and secured environment that could improve referral to PSU. A psychiatrist was the responsible person in this mission, in collaboration with information technologist. The change was tested on Sunday, November 27. The run chart shows the number of new cases distributed in relation to the median and target line, which is measured on a weekly basis. As we can observe, after conducting Plan-Do-Study-Act 2 in week 11, there is an increase in the cases to three cases in that week, exceeding the target line and reflecting the effectiveness of the second change idea by the prediction regarding increasing the number of the referred cases. The results of Plan-Do-Study-Act 2 had encouraged the team to move on and add the change ideas number 3 of re-engineering the PSU process and to be tested in Plan-Do-Study-Act 3 (see more details about PDSA 2 in table 1).

\section{Plan-Do-Study-Act 3}

Re-engineering the referral process to PSU in order to be smoother, short and lean, avoiding sophisticated process. The station manager and team leader were both assigned for this task, which was carried on Sunday, December 4. The run chart shows increase in the number of new cases, which is measured on a weekly basis. As we can observe, after conducting Plan-Do-Study-Act 3 in week 12, there was a negative impact on the number of cases referred to PSU in that week below the target and even the median reaching no cases at that week. Our prediction regarding increase in referred cases was not confirmed. However, after discussion with the team, we realised that some changes such as process re-engineering might take some time due to the latent effect. Therefore, we decided to track the effect over week 13 until 18. Then the number of referred cases started rising again starting from week 13, proving the latent effect that exceeds both the median and target line. Because of the improvement of referral process showed by the new cases referred to PSU following Plan-Do-Study-Act 3, which was measured in the next 6 weeks following Plan-Do-Study-Act 3, we accepted this change (see more details about Plan-DoStudy-Act 3 in table 1).

\section{RESULTS}

The impact of the tested change idea is shown in a run chart after conducting the three Plan-Do-Study-Act cycles. 
Table 1 Different PDSA cycles to test interventions in three successive weeks

$\begin{array}{lll}\text { PDSA cycle } 1 & \text { PDSA cycle 2 } & \text { PDSA cycle } 3\end{array}$

\begin{tabular}{ll}
\hline Aim & $\begin{array}{l}\text { Increasing awareness of PSU } \\
\text { and the services provided using } \\
\text { brochure through emails to all SRCA } \\
\text { employees by } 80 \% \text { in } 1 \text { week }\end{array}$ \\
Plan & $\begin{array}{l}\text { Sending educational materials about } \\
\text { PSU and its services through SRCA } \\
\text { emails for all employees }\end{array}$
\end{tabular}

Prediction We think all SRCA users will receive emails about PSU brochure that will increase awareness about PSU services and subsequently increase the number of referred cases

Do We sent PSU brochures through SRCA emails to the employees to increase awareness about PSU services and subsequently increase the number of referred cases to PSU. Problems: We needed authority from CEO to send the emails to all users through the IT department, and we got that authority.

Feedback: CEO has adopted a unified emailing system called 'internal network' for future emails for all users.

\begin{tabular}{|c|c|}
\hline Study & $\begin{array}{l}\text { The run chart shows the number of } \\
\text { new cases distributed in relation to } \\
\text { the median and target line, which is } \\
\text { measured on a weekly basis. As we } \\
\text { can observe, after conducting PDSA } \\
1 \text { in week } 10 \text {, still the number of } \\
\text { cases was below our target. } \\
\text { Our prediction regarding increase in } \\
\text { referred cases was not confirmed; } \\
\text { we learnt that emails alone could not }\end{array}$ \\
\hline
\end{tabular}

Establishing electronic consultations at PSU in SRCA in a 1-week period in Riyadh

Increasing the number of referred cases to PSU directly by $50 \%$ in a 1-week period in Riyadh

Starting electronic consultation services for SRCA personnel who are seeking mental support

The number of new cases referred would increase due to the privacy of the new channels.

\section{We established electronic} consultation services through SRCA website in order to reduce the effect of one important barrier that prevents employees from seeking PSU services, which is privacy. The psychiatrist is the only person who receives employees' complaint and deals with it in a professional way.

Problems: No specific problems were observed except the increased workload in PSU.

Feedback: The service was welcomed among different SRCA departments.

The run chart shows the number of new cases distributed in relation to the median and target line, which is measured on a weekly basis. As we can observe, after conducting PDSA 2 in week 11, there is an increase of cases to three cases in that week, exceeding the target line. This reflects the effectiveness of the current change.

Our prediction regarding increase in referred cases was confirmed.
Re-engineering the referral process to be directly from the employees to PSU, saving time, effort and confidentiality

The number of new cases referred would increase due to the short and easy route for the new referral process.

The team has changed the old referral process, which was passing through multiple hierarchical levels, consuming a lot of time and may disseminate patient data to others. We came up with a short cut referral route from the patient to the PSU directly.

Problems: No specific problems were noticed.

Feedback: The service was welcomed among different SRCA departments.

The run chart shows the number of new cases distributed in relation to the median, which is measured on a weekly basis. As we can observe, after conducting PDSA 3 in week 12 , there is a negative impact on the number of cases referred to PSU in that week below the target and even the median. Our prediction regarding increase in referred cases was not confirmed. Therefore, we decided to track the effect over weeks 13-18. The number of referred cases started to rise again during weeks 13 and 18 , proving the latent effect.

Because of the improvement of the new cases referred to PSU following PDSA 3, which was measured in the next 6 weeks, we will accept this change.
2 , which is establishing electronic consultation channels for PSU.
Because of the improvement of the new cases referred to PSU, following PDSA 2, we will accept this change, which is establishing electronic consultation channels for PSU. The team preferred moving to PDSA 3 since this change is more important and durable.

PSU, Psychological Support Unit; SRCA, Saudi Red Crescent Authority Executive Officer; IT, Information Technology.

Overall, the number of referral to the PSU at SRCA in Riyadh Region had improved compared with the baseline data. This reflects the effectiveness of using multifaceted approach in bringing about real improvement. As shown in the run chart, over 18 weeks, the number of referred cases started to rise again during week 13 until 18 , showing the impact of the test idea, as shown in the run chart (figure 4). 


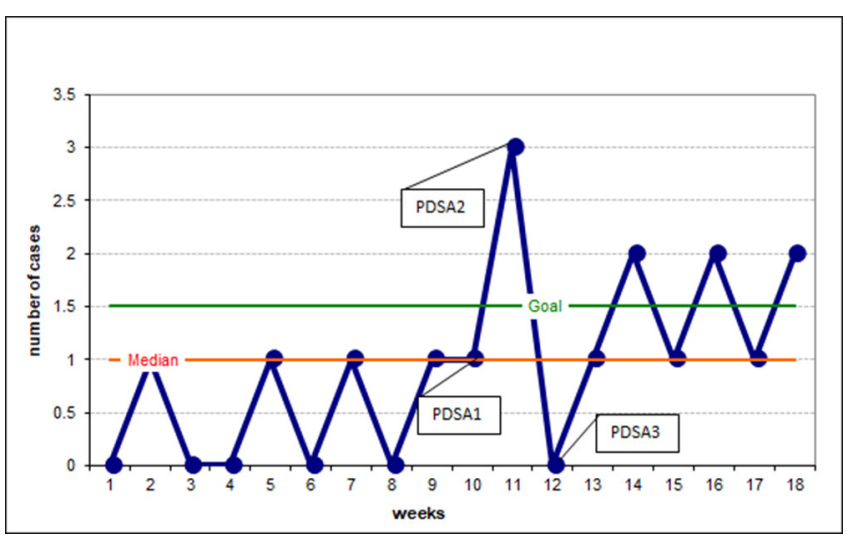

Figure 4 Number of referred cases to Psychological

Support Unit postinterventions. PDSA, Plan-Do-Study-Act.

\section{LESSONS AND LIMITATIONS}

We can summarise lessons learnt as follows: multidisciplinary teamwork from different disciplines and backgrounds is crucial in any quality improvement project. We exposed different phases of teamwork, that is, forming, storming, norming, then performing. The team members managed to work in harmony, knowing each duties and responsibilities. Another lesson is that sending emails only cannot be sufficient to raise awareness, so we might need to have more tools and channels to raise awareness, such as orientation sessions targeting SRCA employees. In quality improvement projects, we should be aware of the negative effect that precedes the latent effect and try to allow more time to produce the positive effect as we found in re-engineering process, that is, negative results sometimes can be the start to pave the road for the positive ones. We learnt also that improvement projects using Plan-Do-Study-Act cycles are flexible for experiencing and learning over time, in which you can amend, adjust or even totally change the whole intervention based on the results yielded. One of the limitations is that the project results represent only one PSU in Riyadh Region, which might affect the generalisability to other PSU in SRCA in different regions in Saudi Arabia and beyond. However, the change ideas and results look promising and worth replicating somewhere else. Another limitation is that we did not use balancing measure to assess the impact of these change ideas on other parts of the system.

\section{CONCLUSION}

We concluded that re-engineering referral process had the most effective impact among other change ideas in terms of magnitude and sustainability of the effect on increasing the number of referral cases to the PSU, and we recommend further testing of these changes in PSU across the SRCA in other regions. However, despite the short effects of electronic consultations for EMS workers, it had good impact, which is aligned with previous study findings that reported a positive impact of using electronic consultation on mental health. ${ }^{3}$ The multifaceted approach is a good strategy, that is, after testing the three designated changes; we found usefulness of the project reflecting an immediate effect on SRCA employees as shown in the increased number of referred cases to PSU. In order to keep these good results of the project, one must think seriously of how to maintain and sustain those changes that must be anchored in the daily practice of system. The modified system will ease the use of the new changes and make it difficult to use the old one. Three important components must be involved to assure sustainability of the projects, namely staff, process and organisation. Staff must undergo a thorough training and development that maintain a good level of awareness about PSU and the new way to reach its services. The process must monitor the progress and characterised by adaptability and credibility of evidence assuring direct referral to PSU. Regarding the third component, the organisation must develop the infrastructure that can easily accommodate the evidence-based improvement changes in a way that fits goals and cultures of our organisation. As one think about spreading and sustainability of this project, it can be spread to other organisations that had the same services provided by PSU. We recommend further test to be carried on in the future for using electronic consultations services for those seeking support, hence it showed some temporal improvements and need to be adapted. Furthermore, another programme must be thought of aiming at raising awareness of existence as well as the services provided by the unit.

Acknowledgements The authors acknowledge the Psychological Support Unit members, EMS department staff in Saudi Red Crescent Authority.

Contributors AYA and TMB have planned this project. SAM performed conduction of the study, while both AYA and SAM reported the results. KA-S and AYA contributed to submitting and reviewing the manuscript as guarantors.

Funding None declared.

Competing interests None declared.

Provenance and peer review Not commissioned; externally peer reviewed.

Open Access This is an Open Access article distributed in accordance with the Creative Commons Attribution Non Commercial (CC BY-NC 4.0) license, which permits others to distribute, remix, adapt, build upon this work non-commercially, and license their derivative works on different terms, provided the original work is properly cited and the use is non-commercial. See: http://creativecommons.org/ licenses/by-nc/4.0/

(c) Published by the BMJ Publishing Group Limited. For permission to use (where not already granted under a licence) please go to http://www.bmj.com/company/ products-services/rights-and-licensing/

\section{REFERENCES}

1. Abbott C, Barber E, Burke B, et al. What's Killing Our Medics?(Ambulance Service Manager Program)4/21/2015. https:/ /static1.squarespace.com/static/555d1154e4b09b430c18fd39/t/ 5599d2b2e4b0c805c287aa3a/1436144306212/What\%27s+Killing+ Our+Medics+Final.pdf

2. Newland C, Barber E, Rose M, et al. CRITICAL STRESS. Survey reveals alarming rates of EMS provider stress \& thoughts of suicide. JEMS 2015;40:30-4.

3. van der Ploeg E, Kleber RJ. Acute and chronic job stressors among ambulance personnel: predictors of health symptoms. Occup Environ Med 2003;60 Suppl 1:40i-6.

4. Bennett P, Williams Y, Page N, et al. Levels of mental health problems among UK emergency ambulance workers. Emerg Med $J$ 2004;21:235-6. 
5. Jalili M, Sadeghipour Roodsari G, Bassir Nia A. Burnout and associated factors among Iranian emergency medicine practitioners. Iran J Public Health 2013;42:1034-42.

6. Patterson PD, Weaver MD, Frank RC, et al. Association between poor sleep, fatigue, and safety outcomes in emergency medical services providers. Prehosp Emerg Care 2012;16:86-97.

7. Ketelaar SM, Nieuwenhuijsen K, Bolier L, et al. Improving work functioning and mental health of health care employees using an e-mental health approach to workers' health surveillance: pretestposttest study. Saf Health Work 2014;5:216-21.

8. Warmerdam L, van Straten A, Twisk J, et al. Internet-based treatment for adults with depressive symptoms: randomized controlled trial. $J$ Med Internet Res 2008;10:e44.

9. National EMS Management Association. Mental health and stress in emergency medicalservices. http://media.cygnus.com/files/base/ EMSR/document/2016/02/Mental_Health_and_Stress.pdf 